\title{
Eficacia del Tratamiento con Amoxicilina en la Prevención de Complicaciones Postoperatorias en Pacientes Sometidos a Cirugía del Tercer Molar: un Estudio Doble Ciego
}

\author{
Efficacy of Amoxicillin Treatment in Preventing Postoperative Complications in Patients \\ Undergoing Third Molar Surgery: a Double Blind Study
}

\begin{abstract}
Walter Leal de Moura*; Weber Leal de Moura*; Simei André da Silva Rodrigues Freire*; Suyá Moura Mendes ${ }^{* * *} \&$ Sergio Olate ${ }^{* * * *}$
\end{abstract}

DE MOURA, W. L.; DE MOURA, W. L.; FREIRE, S. A. S. R.; MENDES, S. M. \& OLATE, S. Eficacia del tratamiento con amoxicilina en la prevención de complicaciones postoperatorias en pacientes sometidos a cirugía del tercer molar: un estudio doble ciego. Int. J. Odontostomat., 5(2):147-152, 2011.

RESUMEN: El objetivo de esta investigación fue estudiar clínicamente la eficacia de la terapia con amoxicilina en terceros molares retenidos sobre las condiciones postoperatorias de edema, dolor, trismus e infección. Fueron seleccionados 14 pacientes de entre 15 y 30 años con la retención de los dientes 3.8 y 4.8 en la misma posición según las clasificaciones utilizadas actualmente. Se realizó inicialmente una cirugía y posteriormente la segunda; se estableció un grupo test con terapia antibiótica (amoxicilina 500mg c/8h por 7 días) y un grupo control que no utilizo antibióticos. El edema fue estudiado a través de la distancia de puntos faciales de referencia y la evaluación del trimus fue obtenida por medio de la distancia interincisal superior e inferior. El dolor fue estudiado mediante escala visual análoga. No existió diferencia estadísticamente significativa en relación al edema y al trismus en los periodos estudiados; existió diferencia estadísticamente significativa respecto al dolor durante los primeros dos días, siendo el grupo test que presentaba más dolor. A los 7 días ya no se apreció diferencias. Finalmente, la amoxicilina como terapia posterior a la exodoncia de terceros molares no se relaciona con las condiciones postoperatorias del paciente

PALABRAS CLAVE: terapia antibiótica, edema, trismus, cirugía del tercer molar.

\section{INTRODUCCIÓN}

Los terceros molares son dientes que frecuentemente se encuentran incluidos (Mercier \& Precious, 1992) posiblemente debido a que son los últimos dientes en completar su formación radicular y las últimas piezas en completar el desarrollo radicular (Garcia, 2000). Aunque las indicaciones de exodoncia son variadas, una de ellas se centra en el hecho de que potencialmente pueden causar patologías complejas y alterar la salud periodontal del segundo molar (Knutsson et al., 1992); en este sentido, la literatura científica parece inclinarse a realizar la exodoncia de terceros molares de forma precoz (hasta los 22 años) para prevenir complicaciones futuras (Mercier \& Precious).
Por otra parte, las infecciones locales y la pericoronaritis son frecuentemente llevadas a la indicación de exodoncia (Bruce et al., 1980; Martin et al., 1997; Peterson, 1992); también lo son la reabsorción radicular de dientes adyacentes, dolor regional asociado al tercer molar, alteración oclusal, fracturas mandibulares con compromiso del tercer molar, entre otros (Osborn et al., 1985).

Los avances tecnológicos nos instalan hoy con técnicas y procesos que optimizan resultados, permitiendo que la cirugía del tercer molar sea segura y eficaz; no obstante, existen potenciales complicaciones como las que aquejan a cualquier tipo de cirugía

Cátedra de Cirurgia e Traumatologia Buco Maxilo Facial de la Universidade Federal do Piauí, Brasil.

* Departamento de Morfologia do Centro de Ciências da Saúde da Universidade Federal do Piauí, Brasil.

** Cirujano Dentista, Bolsista da Universidade Federal do Piauí, Brasil.

**** División de Cirugía Oral y Maxilofacial, Departamento de Odontología Integral, Universidad de La Frontera, Chile. 
DE MOURA, W. L.; DE MOURA, W. L.; FREIRE, S. A. S. R.; MENDES, S. M. \& OLATE, S. Eficacia del tratamiento con amoxicilina en la prevención de complicaciones postoperatorias en pacientes sometidos a cirugía del tercer molar: un estudio doble ciego. Int. J. Odontostomat., 5(2):147-152, 2011.

invasiva (Chiapasco et al., 1993). De esta forma, dentro de la cirugía oral, la cirugía del tercer molar (quizás por ser realizada con más frecuencia que otro tipo de cirugías) es la que causa mayor complicación e incomodidad en el post operatorio; por ejemplo, se encuentra sujeta a la presencia de infección o alveolitis (Indresano et al., 1992), como cualquier otra exodoncia, con la salvedad de que su ubicación anatómica es una de las más complejas dentro de la cavidad oral.

El uso de antibióticos para cirugía del tercer molar retenido es controversial. La incidencia de infecciones post operatorias en estos procedimientos quirúrgicos esta entre el $1 \%$ y el $5 \%$, lo que no justificaría el uso rutinario de antibióticos (Thomas \& Hill, 1997; van Der Zwan et al., 1982; van Gool et al., 1977; Zeitler, 1995). No obstante, existen algunas publicaciones que señalan diferencias estadísticamente significativas entre índices de infección post operatoria en cirugía oral de pacientes tratados con o sin la administración del antibiótico (Eliasson et al., 1989).

Esta investigación tiene por objetivo estudiar clínicamente la eficacia del uso de antibióticos en cirugía de terceros molares inferiores retenidos y su impacto en las condiciones en la primera semana de post operatorio.

\section{MATERIAL Y MÉTODO}

Muestra. Se seleccionaron 14 pacientes de entre 15 y 30 años para extraer el tercer molar inferior retenido sin distinción de género o raza; los sujetos fueron seleccionados de aquellos pacientes con indicación de exodoncia de tercer molar (indicación realizada por un odontólogo experimentado), en la Clínica de Odontología de la Universidad Federal de Piauí, Brasil. Dentro de los criterios de inclusión se consideró la indicación de exodoncia de ambos terceros molares inferiores con una posición semejante según la clasificación de Pell y Gregory y la clasificación de Winter; las cirugías debían ser totalmente electivas y sin ningún indicio de patología infecciosa o traumática en relación al tercer molar; como criterio de exclusión estaba el consumo de tabaco dentro de los últimos 12 meses y la adicción al alcohol considerada como la ingesta de alcohol por más de 2 veces a la semana. Todos los pacientes accedieron voluntariamente al estudio siendo aprobado por el comité de ética de la Universidad Federal de Piauí.
Protocolo quirúrgico. Todos los procedimientos quirúrgicos fueron desarrollados por un solo operador, siendo realizada en una primera etapa la cirugía de un molar (3.8 o 4.8) y luego de mínimo 4 semanas, era realizada la cirugía para extraer el diente contralateral. El operador mantuvo el mismo colgajo para ambas cirugías mediante el uso de una hoja de bisturí № 15; cuando fue necesaria ostectomía y/o odontosección, esta se realizó con pieza de alta rotación (400.000 rpm), fresa del tipo tronco cónica № 703 e irrigación de suero fisiológico al 0,9\%; las técnicas de sutura fueron abordadas según el colgajo realizado mediante hilo de seda 3-0 montada en aguja de $18 \mathrm{~mm}$ en $3 / 4$ de ciclo y compresión final con gasa durante 30 minutos posteriores a la cirugía. En la etapa de post operatorio inmediato se determinó el tiempo quirúrgico (desde el inicio del acto quirúrgico hasta el la instalación de la última sutura); todos estos elementos fueron registrando en una ficha clínica especialmente diseñada para este estudio a fin de permitir que la segunda cirugía fuese realizada bajo las mismas condiciones.

Protocolo farmacológico. En todos los pacientes tratados fue empleada la betametasona $4 \mathrm{mg}$ en la etapa de pre operatorio y post operatorio; también se empleó $750 \mathrm{mg}$ de paracetamol cada $6 \mathrm{~h}$ por $48 \mathrm{~h}$. Ambos fármacos fueron administrados por vía oral. Los sitios quirúrgicos fueron escogidos aleatoriamente para determinar la indicación de amoxicilina (500mg cada 8 h por siete días), que comenzó en el momento en que inicio el procedimiento quirúrgico, (vía oral); esto permitió identificar dos grupos de trabajo siendo el grupo 1 aquel sitio quirúrgico donde no se utilizó ningún tipo de antibiótico y el grupo 2 aquel sitio quirúrgico donde se utilizó el esquema antibiótico citado; de esta forma, un paciente pertenece a ambos grupos en diferentes momentos (intervención inicial o final).

Estudio y control postoperatorio. Los pacientes fueron controlados diariamente durante una semana, estudiándose la temperatura corporal tres veces al día; dentro de los criterios de seguimiento, si el paciente presentara sobre $37,8^{\circ} \mathrm{C}$ oral, dolor intenso o supuración activa sería sometido a una rigurosa evaluación clínica para identificar el protocolo de tratamiento.

El edema facial fue establecido a través de mediciones realizadas con hilo mediante identificación de puntos de referencia facial; tales puntos fueron ángulo mandibular, tragus, comisura labial, ala de la nariz y canto palpebral lateral; el hilo instalado en un punto facial inicial y terminal determinaba la distancia. Se realizó la medición inicial previo a la cirugía y posterior a la cirugía (según los tiempos de evaluación). 
El trismus fue evaluado mediante la medición de la distancia inter incisal de incisivos superiores e inferiores a fin de identificar el grado de abertura bucal (la medición inicial fue realizada previo al comienzo de la cirugia). La presencia de dolor fue estudiado mediante la escala visual análoga, siendo el paciente responsable de identificar el grado de dolor entre un mínimo de 0 y un máximo de 10.

Todas estas evaluaciones fueron realizadas a las $24 \mathrm{~h}$ de realizada la cirugía, a las $48 \mathrm{~h}$ de la misma y al séptimo día 7 posterior a la cirugía.

Análisis de datos. Se confecciono una base de datos con toda la información recolectada; en el software estadístico Stata $9.0 \otimes$ se realizaron las pruebas estadísticas t de Student, test de Wilcoxon y el test de Señales considerando el valor de $p<0,05$ para obtener relación estadísticamente significativa.

\section{RESULTADOS}

En ninguna de las evaluaciones realizadas en ambos grupos se identificó ningún signo o síntoma de infección postoperatoria del sitio quirúrgico; Mediante la prueba t de Student se evaluaron las variables asociadas al edema facial como la distancia entre ángulo mandibular y los diferentes puntos de referencia obteniéndose ausencia de diferencias estadísticamente significativa (Tabla I) entre el grupo 1 y grupo 2 .

Tabla I. Comparación de las medidas obtenidas en las evaluaciones de los 14 pacientes en las 28 cirugías realizadas según el valor de $p$ (prueba t de Student) según el grupo de aplicación de amoxicilina.

\begin{tabular}{lccc}
\hline Relación & $\mathbf{2 4 h}$ & $\mathbf{4 8 h}$ & $\mathbf{7}$ Días \\
\hline D AM-T & 0,340 & 0,686 & 0,088 \\
D AM-E & 0,549 & 0,497 & 0,549 \\
D AM-AN & 0,864 & 0,863 & 0,909 \\
D AM-Q & 0,344 & 0,641 & 0,817 \\
D AM-M & 0,946 & 0,474 & 0,574 \\
D II & 0,539 & 0,536 & 0,537 \\
Dolor & $0,044^{*}$ & $0,024^{*}$ & 0,180 \\
\hline
\end{tabular}

D AM-T: Distancia ángulo mandibular - tragus; D AM-E: Distancia ángulo mandibular - exocantion; D AM-AN: Distancia ángulo mandibular - ala lateral de la nariz; D AM-Q: Distancia ángulo mandibular - queilon; D AM-M: Distancia ángulo mandibular - mentón; D II: Distancia interincisal. *Estadisticamente significativo.
El dolor fue estudiado con el test de Wilcoxon en los diferentes periodos de evaluación y se obtuvo un valor de $p=0,024$ considerando las mediciones realizadas a las 24 y $48 \mathrm{~h}$, indicando diferencia estadísticamente significativa favorable al empleo de antibióticos; sin embargo, en la evaluación realizada en el día 7 de postoperatorio no existió diferencias presentando un valor de $p=0,180$.

\section{DISCUSIÓN}

La cirugía oral ha evolucionado y desarrollado en diferente condiciones, siendo una necesidad fundamental la de realizar los procedimiento en una cadena aséptica que asegura la esterilidad de los instrumentos utilizados; el empleo de antibióticos muchas veces vino a suplir la deficiencia de estos protocolos de forma que cuando se comparaba su efectividad, en algunos casos prevenía infecciones mientras que en otros su utilidad era cuestionada (Castro, 1998).

La infección se desarrolla cuando existe una agresión bacteriana cuantitativa y cualitativamente importante. Se desarrolla con mayor frecuencia en pacientes cuyos mecanismos de defensa están debilitados; visualizando este concepto, la prevención se basa en la reducción del número de bacterias en la herida quirúrgica y en la mejoría de la capacidad inmunológica del paciente (Peterson). El riesgo de infección varía, entonces, de acuerdo al tipo de procedimiento y a las condiciones del paciente (edad por ejemplo), presencia de obesidad, diabetes descompensada, inmunodepresión, presencia de infección activa y tiempo quirúrgico (Sands et al., 1993a, 1993b).

La aplicación de antibióticos en pacientes sometidos a cirugía oral es controversial; una parte importante de los autores no indica su uso "preventivo" ya que en estudios con uso de placebo se han obtenidos resultados similares a los obtenidos en esta investigación (Happonen et al., 1990; Zeitler). Otro punto de evaluación está en la generación de resistencias bacterianas, que en algunos casos puede presentar importantes consecuencias individuales y poblacionales (van der Swan et al.; Zeitler) además de las propias complicaciones y efectos colaterales de fármacos de este tipo. No obstante, es claro que en casos donde existe una infección establecida asociada al tercer molar, un correcto tratamiento sugiere el uso de antibioterapia previa al procedimiento quirúrgico (Piecuch et al., 1995). 
DE MOURA, W. L.; DE MOURA, W. L.; FREIRE, S. A. S. R.; MENDES, S. M. \& OLATE, S. Eficacia del tratamiento con amoxicilina en la prevención de complicaciones postoperatorias en pacientes sometidos a cirugía del tercer molar: un estudio doble ciego. Int. J. Odontostomat., 5(2):147-152, 2011.

De esta forma podemos reconocer la profilaxis antibiótica que se indica a pacientes según la norma de la AHA (American Heart Association) y podemos reconocer el tratamiento antibiótico empleado como esquema terapéutico ante la infección activa (Andrade et al., 1998); bajo este concepto, cabe someter una pregunta de análisis como ¿en cuál de estas dos categorías figura el uso de antibióticos en un paciente sometido a cirugía de exodoncia de tercer molar con técnica aséptica y que no presenta infección inicial?; la respuesta es compleja debido a que la naturaleza empírica y la ciencia se confunden en este punto, impidiendo juicios críticos sean basados en evidencia por parte de algunos clínicos sometidos a la ejecución de estas técnicas (Palmer, 2003); no obstante, la respuesta de la literatura científica, confirmado en esta investigación, nos lleva a señalar que no es necesario indicar terapia o profilaxis antibiótica en estos pacientes (Harrison \& Svect,1998). En la misma dirección, otros estudios han señalado que el no indicar antibioterapia en estos casos permite disminuir los costos económicos y biológicos del procedimiento (Thomas \& Hill).

Las cirugías realizadas en esta investigación contemplaron centro quirúrgico y cadena aséptica. En ninguno de los dos grupos se observó presencia de infección en el sitio quirúrgico. A pesar de que la alveolitis no tiene directa relación con infección del sitio quirúrgico (Krekmanov, 1981), tampoco fue observada en ninguno de los 14 sitios quirúrgicos.

El edema facial y el trismus asociados a la exodoncia del tercer molar incluido son frecuentes y responden a las condiciones anatómicas y fisiológicas de la región y son consecuencia de la etapa de reparación de tejidos ya que existe una destrucción inicial de vasos sanguíneos, linfáticos, tejido muscular, entre otros que hacen que el edema pueda ser más o menos expresivo (Lopez et al., 1995). Diversos métodos de estudio del edema facial han sido propuestos aunque ninguno ha sido utilizado protocolarmente de forma simple y fidedigna (van Gool et al.); no obstante, parece que la adopción de puntos faciales (definidos por cada autor en particular) son los elementos más utilizados en la literatura y apoya su empleo en esta investigación (Amin \& Laskin, 1983). Mediante esta metodología, el edema facial observado en esta muestra no presentó diferencias significativas entre ambos grupos.

Para el estudio del dolor, la escala visual análoga ha sido empleada históricamente y representa un patrón seguido por muchas y variadas investigaciones; si bien el control del dolor se realiza con analgesia (Dionne et al., 1984), algunos clínicos sugieren que el hecho de aplicar antibioterapia y "disminuir el riesgo de infección” también induce la reducción en la percepción del dolor. En nuestra investigación existió diferencia en la percepción del dolor durante los primeros dos días mientras que en la evaluación de $7^{\circ}$ día no existió ningún tipo de diferencia entre ambos grupos.

En base a los resultados de este trabajo y considerando las limitaciones que impone nuestra reducida muestra, podemos señalar que los antibióticos aplicados en pacientes asintomáticos no se relaciona con disminución del edema, disminución del dolor, disminución del trismus o limitación de la infección y que cuando se respeta la cadena aséptica y las normas de bioseguridad, es posible efectuar estas cirugías con limitados riesgos de infección y complicaciones post operatoria.

DE MOURA, W. L.; DE MOURA, W. L.; FREIRE, S. A. S. R.; MENDES, S. M. \& OLATE, S. Efficacy of amoxicillin treatment in preventing postoperative complications in patients undergoing third molar surgery: a double blind study. Int. J. Odontostomat., 5(2):147-152, 2011.

ABSTRACT: The aim of this study was to clinically evaluate the efficacy of antibiotics in retained third molar extractions, determining the need of antibiotics prophylaxis use in these procedures. Fourteen (14) patients, aged between 15 and 30 years, irrespective of sex, color or race were selected, for removal of retained third molars in symmetrical positions. The surgeries were divided into two groups: the control group in the first surgery and the second group undergoing prophylactic administration of $500 \mathrm{mg}$ of amoxicillin 8 / 8 hours, for seven days after surgery. The evaluation of edema was established by the distance of reference points and assessment of facial trismus and was obtained by top and bottom interincisal distance. Measurements were performed before and after surgery, 24, 48 hours and for 7 days. Pain was evaluated subjectively by a visual analog scale. There was no statistically significant difference with respect to swelling and trismus after dental extractions with or without the use of antibiotics, and there was statistically significant difference in relation to pain in the postoperative periods of 24 and 48 hours, and increased pain reported by the test group. At day seven no differences were noted in perception of pain between the groups. Finally, the amoxicillin therapy subsequent to the third molar surgery is not related to the postoperatory conditions of the patient.

KEY WORDS: antibiotic therapy, edema, trismus, third molar surgery. 
DE MOURA, W. L.; DE MOURA, W. L.; FREIRE, S. A. S. R.; MENDES, S. M. \& OLATE, S. Eficacia del tratamiento con amoxicilina en la prevención de complicaciones postoperatorias en pacientes sometidos a cirugía del tercer molar: un estudio doble ciego. Int. J. Odontostomat., 5(2):147-152, 2011.

\section{REFERENCIAS BIBLIOGRÁFICAS}

Amin, M. M. \& Laskin, D. M. Prophylatic use of indomethacin for prevention of post surgical complications after removal of impacted third molar. Oral Surg. Oral Med. Oral Pathol. Oral Radiol. Endodont., 55(5):448-51, 1983.

Andrade, E. D.; Passeri, L. A. \& Mattos Filho, T. R. Prevenção da endocardite bacteriana: novas recomendações da American Heart Association. Rev. Assoc. Paul. Cir. Dent., 52(5):353-7, 1998.

Bruce, R. A.; Frederickson, G. C. \& Small, G. S. Age of patients and morbidity associated with mandibular third molar surgery. J. Am. Dent. Assoc., 101(2):240-5, 1980.

Castro, W. H. Antibiótico profilático em cirurgia bucomaxilo-facial. Revista do C. R. O. M. G., 4:46-53, 1998.

Chiapasco, M.; De Cicco, L. \& Marrone, G. Side effects and complications associated with third molar surgery. Oral. Surg. Oral Med. Oral Pathol., 76(4):412-20, 1993.

Dionne, R. A.; Wirdzek, P. R.; Fox, P. C. \& Dubner, R. Supression of postoperative pain by the combination of a nonsteroidal anti-inflammatory drug, flurbiprofen, and a long-acting local anesthetic, etidocaine. J. Am. Dent. Assoc., 108(4):596-601, 1984.

Eliasson, S.; Heimdahl, A. \& Nordenram, A. Pathological changes related to long term impaction of third molar. Int. J. Oral Maxillofac. Surg., 18(4):210-2, 1989.

Garcia, R. R.; Paza, A. O.; Moreira, R. W. F.; de Moraes, M. \& Passeri, L. A. Avaliação radiográfica da posição de terceiros molares inferiores segundo a classificação de Pell \& Gregory e Winter. $R$. F. O., 5(2):31-6, 2000.

Happonen, R. P.; Bäckström, A. C. \& Ylipaavalniemi, P. Prophylactic use of phenoxymethylpenicillin and tinidazole in mandibular third molar surgery, a comparative placebo controlled clinical trial. Br. J. Oral Maxillofac. Surg., 28(1):12-5, 1990.

Harrison, J. W. \& Svect, T. A. The benning of the end of the antibiotic era? Part I. Abuse of miracle drugs. Quintessence Int., 29(3):151-62, 1998.

Indressano, A. T.; Haug, R. H. \& Hoffman, M. J. The third molar as a cause of deep space infections. J. Oral. Maxilofac. Surg., 50(1):33-6, 1992.

Knutsson, K.; Brehmer, B.; Lysell, L. \& Rohlin, M. Asymptomatic mandibular third molars: oral surgeons' judgment of the need for extraction. $J$. Oral Maxillofac. Surg., 50(4):329-33, 1992.

Krekmanov, L. Alveolitis after operative removal of third molars in the mandible. Int. J. Oral Surg., 10(3):173-9 1981.

Lopez, V.; Mumenya, R.; Feimann, C. \& Harris, M. Third molar surgery: an audit of the indicators for surgery, postoperative complaints and patient satisfaction. Br. J. Oral Maxillofac. Surg., 33(1):335, 1995.

Martin, M. V.; Longman, L. P.; Hill, J. B. \& Hardy, P. Acute dentoalveolar infections: an investigation of the duration of antibiotic therapy. Br. Dent. J., 183(4):135-7, 1997.

Mercier, P. \& Precious, D. Risks and benefits of removal of impacted third molars. A critical review of the literature. Int. J. Oral Maxillofac. Surg., 21(1):17-27, 1992.

Osborn, T. P.; Frederickson, G. Jr.; Small, I. A. \& Torgerson, T. S. A prospective study of complications related to mandibular third molar surgery. J. Oral. Maxillofac. Surg., 43(10):767-9, 1985.

Palmer, N. A. O. Revisiting the role of dentists in prescribing antibiotics. Dent Update, 30(10):5704, 2003.

Peterson, L. J. Rationale for removing impacted teeth: when to extract or not extract. J. Am. Dent. Assoc., 123(7):198-204, 1992.

Piecuch, J. F.; Arzadon, J. \& Lieblich, S. E. Prophylatic antibiotics for third molar surgery: a supportive opinion. J. Oral Maxillofac. Surg., 53(1):53-60, 1995. 
DE MOURA, W. L.; DE MOURA, W. L.; FREIRE, S. A. S. R.; MENDES, S. M. \& OLATE, S. Eficacia del tratamiento con amoxicilina en la prevención de complicaciones postoperatorias en pacientes sometidos a cirugía del tercer molar: un estudio doble ciego. Int. J. Odontostomat., 5(2):147-152, 2011.

Sands, T.; Pynn, B. R. \& Nenniger, S. Third molar surgery: current concepts and controversies. Part 1. Oral Health, 83(5):11-4, $1993 a$.

Sands, T.; Pynn, B. R. \& Nenniger, S. Third molar surgery: current concepts and controversies. Part 2. Oral Health, 83(5):19, 21-2, 27-30, 1993b.

Thomas, D. W. \& Hill, C. M. An audit of antibiotic prescribing in third molar surgery. Br. J. Oral Maxillofac. Surg., 35(2):126-8, 1997.

van del Zwan, J.; Boering, G.; Wesseling, H.; Smit Sibinga, C. T. \& van der Weele, L. T. The lower third molar and antiphlogistics. Int. J. Oral Maxillofac. Surg., 11(6):340-50, 1982.

van Gool, A. V.; Ten Bosch, J. J. \& Boering, G. Clinical consequences of complaints and complications after removal of the mandibular third molars. Int. J. Oral Surg., 6(1):29-37, 1977.

Zeitler, D. L. Prophylactic antibiotics for third molar surgery: a dissenting opinion. J. Oral Maxillofac. Surg., 53(1):61-4, 1995.
Dirección de correspondencia:

Prof. Dr. Walter Leal de Moura Cátedra de Cirurgia e Traumatologia

Buco Maxilo Facial

Universidade Federal do Piauí

Rua São Pedro, 3274/902-Ilhotas

Cep: 64004-260

Teresina-PI

BRASIL

Email: centroface@uol.com.br

Recibido : 05-06-2011

Aceptado: 19-07-2011 